\title{
CONCEPCIONES ALTERNATIVAS SOBRE EL CAMPO MAGNETICO ESTACIONARIO. SELECCIÓN DE CUESTIONES REALIZADAS PARA SU DETECCIÓN
}

\author{
GUISASOLA, JENARO, ALMUDÍ, JOSÉ MANUEL y CEBERIO, MIKEL \\ Departamento de Física Aplicada I. Universidad del País Vasco - Euskal Herriko Unibertsitatea
}

\begin{abstract}
Resumen. El trabajo que aquí se presenta trata de hacer una recapitulación de las principales investigaciones realizadas en el ámbito del campo magnético estacionario, que tienen como objetivo averiguar las deficiencias más importantes que se producen en el aprendizaje de los conceptos inherentes al área de la física citada. Esta selección de trabajos, esperamos que sirvan para que el profesorado interesado por el tema pueda hacer una primera aproximación a las concepciones más problemáticas que tienen los estudiantes o, en todo caso, a aquéllas que han sido investigadas en el contexto de la didáctica de la física.
\end{abstract}

Palabras clave. Magnetismo, concepciones alternativas, dificultades de aprendizaje, revisión bibliográfica, implicaciones didácticas.

Summary. The work we present here is an attempt to recapitulate the main research studies carried out within the field of stationary magnetic field. The objective of these studies is to find out the main deficiencies produced in learning the concepts inherent to the mentioned area of Physics. We expect this collection of studies will help those teachers interested in the subject in their first approach to students problematic conceptions or, at least, to those problematic conceptions studied within the context of Physics Education. Keywords. Magnetism, alternative conceptions, learning difficulties, bibliographic review, consequences for teaching.

\section{INTRODUCCIÓN}

El papel y las funciones que se atribuyen a los estudiantes en el proceso educativo han cambiado durante las últimas décadas. La orientación constructivista del aprendizaje considera que los estudiantes construyen sus propios conocimientos mediante la interacción de sus estructuras mentales con la información que reciben del exterior (Driver, 1989; Driver et al., 1985). Cuando los estudiantes escuchan una conferencia, leen el libro de texto u observan un hecho físico, interpretan esa información sobre la base de la estructura del conocimiento que ya poseen; ésta, con frecuencia, incluye conceptos intuitivos o esquemas alternativos que han demostrado ser muy resistentes al cambio. Esto ha llevado a dar una importancia a los conocimientos previos y a las estructuras mentales de los estu- diantes que no era reconocida en otras teorías del aprendizaje. En este sentido, en las últimas décadas se han publicado numerosos trabajos de investigación de ámbito internacional que caracterizan los procesos de conocimiento y pensamiento alternativos o iniciales que los estudiantes traen consigo a la enseñanza de las ciencias. Muestra de ello es la extensa recopilación bibliográfica sobre este tipo de investigaciones editada por Pfundt y Duit (1998, 6a. edición). En particular, el $61 \%$ de las investigaciones referenciadas corresponde al área de física.

Muchas de las investigaciones se han centrado en las diferencias entre lo que los estudiantes aprenden y lo que el profesor enseña. Estas desviaciones de los estudiantes res- 
pecto a la teoría científica aceptada se suelen denominar concepciones alternativas (Wandersee et al., 1994). El constructivismo considera que estas «concepciones alternativas» son productos del proceso de aprendizaje individual de los estudiantes, de su esfuerzo intelectual por dar sentido y organizar una visión del mundo. Sin embargo, es necesario resaltar que estas investigaciones no tratan sólo de detectar el hecho, bien conocido por los profesores, de que los estudiantes no aprenden determinados conceptos y teorías, sino que tratan de caracterizar estas concepciones alternativas y sus posibles causas. La importancia de este análisis de las concepciones y sus causas radica en que podría dar pistas sobre posibles estrategias de enseñanza para superar el poco aprendizaje logrado por los estudiantes (Millar, 1996).

En el área de la física se han tratado ampliamente temas relacionados con la mecánica (Carrascosa y Gil, 1992), así como aquéllos relacionados con circuitos eléctricos elementales (Duit y Von Rhöneck, 1998) y, más recientemente, sobre electrostática (Furió y Guisasola, 1999). Sin embargo, son escasas las investigaciones realizadas en el ámbito del magnetismo. La mayoría de ellas se han desarrollado en los niveles de enseñanza primaria y secundaria, apareciendo muy recientemente (a mediados de la década de los noventa) trabajos en el nivel universitario.

A pesar de la escasez de trabajos que acabamos de reseñar, creemos que el concepto de campo magnético y su fuente constituyen cuestiones básicas sin las que no se puede construir una teoría científica de los fenómenos electromagnéticos. Así pues, nos ha parecido de interés dar una idea global de las aportaciones que han realizado las diversas investigaciones acerca de las ideas de los estudiantes y sus dificultades en el ámbito del campo magnético estacionario.

En física, el estudio del magnetismo es muy amplio e incluye diferentes temas, como propiedades y producción del campo magnético, inducción magnética y radiación electromagnética. En este sentido, en la revisión realizada nos hemos limitado a aquellos estudios que tratan sobre conceptos referidos a campos magnéticos estacionarios producidos por cargas en movimiento con velocidad constante $\mathrm{o}$, de forma equivalente, por corrientes estacionarias dentro de la teoría clásica del electromagnetismo definida por las ecuaciones de Maxwell.

El objetivo que perseguimos es que, dada la importancia de todos los aspectos relacionados con el campo electromagnético, los profesores y profesoras puedan tener unos primeros datos sobre las concepciones alternativas más problemáticas de los estudiantes o, en su defecto, aquéllas que más se han investigado, relacionadas con aspectos inherentes al campo magnético estacionario.

Dado que en los diversos trabajos de investigación analizados se han abordado diferentes aspectos del magnetismo, hemos considerado oportuno agrupar las cuestiones en función de un esquema que iremos desgranando en los siguientes apartados. Sin embargo, es necesario constatar que en la mayor parte de las ocasiones se trata de aspectos relacionados, por lo que recomendamos que no se pierda la visión de conjunto. En todo caso, si hemos optado por realizar esa agrupación de las diferentes cuestiones, a veces no exenta de cierta arbitrariedad, dado el carácter subjetivo de la misma, es porque hemos preferido hacer esta presentación frente a aquella otra que vendría caracterizada por un aborde de las cuestiones de forma desestructurada, la cual podría hacer pensar a los lectores que las concepciones alternativas de los estudiantes son ideas inconexas y sueltas en contra de lo establecido por diferentes investigaciones, según las cuales dichas ideas estarían dotadas de cierta coherencia interna y estructuradas en auténticos «marcos conceptuales alternativos» (Gianneto et al., 1992; Pozo, 1999).

Vamos a continuación a explicitar un resumen de los diferentes trabajos analizados. Para ello cada cuestión se presentará con su enunciado así como su correspondiente referencia (lugar donde es posible localizarla); inmediatamente después se expondrán los comentarios sobre dicha cuestión y, en ocasiones, los resultados obtenidos a la hora de aplicarlas a los grupos de estudiantes.

\section{DIFICULTADES DE APRENDIZAJE DEL CONCEPTO DE CAMPO MAGNETICO Y SUS FUENTES}

En este apartado haremos referencia a diferentes cuestiones que tienen como meta investigar las dificultades relacionadas con el aprendizaje no comprensivo del campo magnético estacionario y sus fuentes. Para ello nos ha parecido oportuno dividir este apartado en otros más concretos:

1.a. Concepciones de los estudiantes sobre la fuente del campo magnético

1.b. Concepciones de los estudiantes sobre el imán como fuente del campo magnético

1.c. Dificultades de aprendizaje respecto a la relación de equivalencia entre una espira de corriente y un imán de acuerdo con el marco teórico de la física

1.d. Dificultades de aprendizaje en la comprensión de la definición operativa del campo magnético

\section{1.a. Concepciones de los estudiantes sobre la fuente del campo magnético}

La línea de trazos de la figura representa la dirección del campo magnético terrestre. En el punto $A$ hay una brújula y en el punto $B$ se encuentra una partícula en reposo cargada con una carga $Q$ positiva.

a) Dibuja sobre $A$ una flecha que represente la orientación de la aguja de la brújula.

b) Explica las razones de tu respuesta.

Referencia: Guisasola, Almudí y Zubimendi (2003)

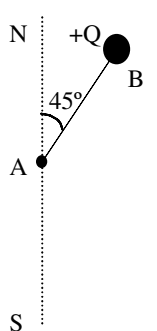


Comentario: Para contestar correctamente a la cuestión bastaría decir que la aguja no experimentará variación en su dirección, ya que las cargas en reposo no generan campo magnético alguno y, por lo tanto, sólo estará sometida al campo magnético terrestre. Sin embargo, sólo el 33\% de los estudiantes de $2^{\circ}$ de bachillerato, el $17 \%$ de media de los de $1^{\circ}$ y $2^{\circ}$ de Ingeniería Técnica Industrial y el 55\% de los de $3^{\circ}$ de Físicas responde correctamente. La mayoría de los estudiantes se encontraría en lo que los autores denominan categoría eléctrica, dentro de la cual se constata una diversificación de argumentos; pero todos ellos coinciden en considerar como fuente del campo magnético las cargas eléctricas en reposo.

Por otro lado, conviene resaltar que un alto porcentaje de respuestas pertenecientes a la categoría aludida considera la interacción magnética como una fuerza central de igual dirección y sentido que la eléctrica. Todos estos resultados sugieren que los estudiantes no identifican correctamente las fuentes del campo magnético y que además muchos de ellos tienen dificultades para distinguir la interacción eléctrica de la magnética.

\section{1.b. Concepciones de los estudiantes sobre el imán como fuente del campo magnético}
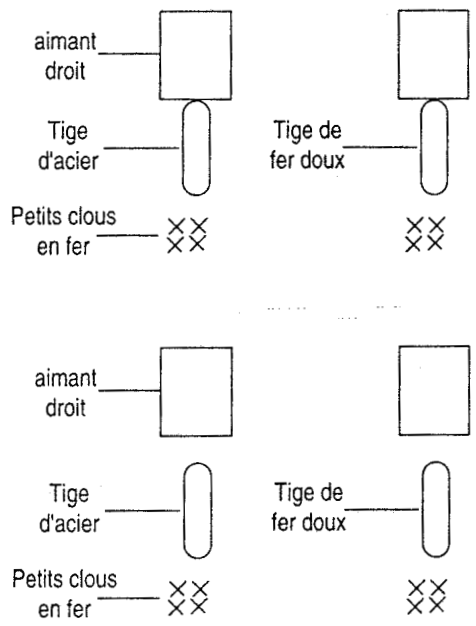

1.b.1. Ponemos en contacto, respectivamente, cada uno de los dos clips (uno de acero y otro de hierro dulce con sendos imanes, y aproximamos unos clavos pequeños a los dos clips (ver figura). Describe lo que observas y trata de explicarlo. Luego retiramos los imanes del lugar donde están los clips, mira qué sucede e intenta dar una explicación.

Referencia: Maarouf y Benyamna (1997)

Comentario: Aunque la cuestión es doble, nos interesa destacar en este caso los resultados de la primera parte, puesto que la segunda tiene que ver más con la diferenciación entre materiales ferromagnéticos «duros y blandos».
Respecto de la primera, el asunto que analizaremos es por qué el imán imanta los clips; en definitiva, cómo se entiende que un imán crea un campo magnético. La cuestión fue contestada por 40 estudiantes marroquíes, repartidos en cuatro grupos con 10 estudiantes cada uno de ellos, de edades comprendidas entre los 13 y los 20 años.

Del análisis de las respuestas, los autores deducen que los estudiantes presentan una representación mental que denominan paso de alguna cosa, según la cual la imanación se produce porque algo pasa de «un agente» a «un paciente». Esta representación aparece en el discurso de los cuatro grupos. Sin embargo, las propiedades atribuidas al mediador cambian de un grupo a otro y a veces de un alumno a otro dentro de un mismo grupo. Así, 9 alumnos del primer grupo consideran al mediador como algo abstracto y utilizan nociones como fuerza y energía. Los estudiantes del grupo segundo y una tercera parte del grupo tercero consideran el mediador de la misma manera que los del grupo primero pero utilizando otras denominaciones como: propiedad del imán, imantación, etc. Por contra, 4 de los alumnos del grupo segundo identifican al mediador como una forma de materia: «electrones», «iones», «constituyentes», «materia magnética». Según estos estudiantes, el imán está constituido de una materia que tiene propiedades que le permiten atraer ciertos materiales. Por otro lado, 5 estudiantes del grupo tercero y los del cuarto grupo atribuyen también un aspecto material al mediador identificándolo con portadores de cargas eléctricas; así, cuando el imán se pone en contacto con los clips, pasan cargas del uno al otro de manera que del imán pasan cargas positivas al clip y de éste, negativas al imán, por lo que se atraerán al estar cargados de forma distinta.

En definitiva, los resultados de esta cuestión parecen indicar que los estudiantes entienden que el imán es fuente del campo magnético en función de la propia naturaleza de imán, o bien que los cuerpos que interactúan lo hacen debido a la diferente carga electrostática que tienen. La respuesta correcta considera que los clips (materiales ferromagnéticos) están sometidos al campo magnético del imán, que hará que sus «dominios» se orienten en la dirección y sentido del campo externo, lo que producirá que el propio clip cree su propio campo magnético y actúe sobre los clavos de hierro (material ferromagnético) como si fuera un imán, atrayéndolos.

También hemos analizado alguna otra cuestión (Anexo, cuestión I), en la que los resultados apoyan lo que aquí acabamos de señalar.

1.b.2. Una pequeña muestra de diferentes materiales (cobre, clip de acero, aluminio, latón, madera, caucho, poliestireno, PVC) se coloca enfrente del entrevistado, al que se le hacen diferentes preguntas, como las siguientes:

a) ¿Qué les sucederá a esos objetos cuando se los coloca cerca de un imán?

b) ¿Por qué alguno de esos objetos son atraídos por el imán?

c) ¿Cómo puede actuar un imán incluso a distancia?

d) ¿Por qué el imán se comporta de esa manera?

Referencia: Borges y Gilbert (1998) 


\section{INVESTIGACIÓN DIDÁCTICA}

Comentario: Estas cuestiones se plantean dentro de un estudio realizado a una muestra de 56 personas: estudiantes de edades entre los 15 y los 18 años, profesores de física de secundaria, técnicos en electricidad e ingenieros eléctricos.

Analizadas las respuestas, los autores las agrupan en diferentes modelos:

Modelo A: «Magnetismo como atracción». Según este modelo, el magnetismo es visto como una atracción en una región alrededor del imán y se debe a una propiedad intrínseca de los imanes; es decir, los imanes atraen a algunos materiales a causa de su propia naturaleza y no parece necesaria ninguna explicación añadida.

Modelo B: «Magnetismo como una nube». En este modelo, el imán estaría rodeado por una región limitada dentro de la cual se actuaría sobre otros cuerpos; los fenómenos magnéticos son explicados en términos de la acción de esta región y no de la acción del imán sobre los objetos. Este tipo de concepción parece que proviene de hacer una analogía entre la acción magnética y la gravitatoria.

Modelo C: «Magnetismo como electricidad». Este modelo incluye un mecanismo para explicar la interacción magnética que está basada en la idea de interacción eléctrica. En ese sentido, la atracción magnética, por ejemplo, es vista como la atracción entre cargas de diferente signo, no existiendo una conexión entre atracción y la noción de campo magnético. Los polos de un imán son vistos como regiones que tienen exceso o déficit de cargas eléctricas (concretamente cargas positivas, el polo Norte, y negativas, el polo Sur).

Modelo D: «Magnetismo como polarización eléctrica». Este modelo es una sofisticación del anterior (encontrado entre los estudiantes de alto nivel y también entre alguno de los profesores e ingenieros). El imán polarizaría los objetos cercanos en el sentido eléctrico del término y luego interaccionaría con ellos de forma electrostática.

Modelo E: «Modelo de campo». En este caso, que aparece fundamentalmente entre profesores e ingenieros, se reconocería que los «electrones giratorios» del imán crearían campos magnéticos muy pequeños que al sumarse darían lugar a un campo magnético importante a nivel macroscópico, que actuaría a través de la fuerza magnética sobre las cargas en movimiento.

Se constata que la mayoría de los estudiantes de todos los niveles, técnicos y un porcentaje no despreciable de profesores de secundaria e ingenieros, estarían encuadrados en los modelos A-D.

En otra cuestión que hemos estudiado (Anexo, cuestión II), con un grupo de estudiantes de 9 a 14 años, emergieron categorías que el autor denominaba, pulling model, emanating model y enclosing model que serían coherentes con los modelos A y B aquí presentados.

Los lectores también podrán encontrar trabajos relacionados con los imanes pero que no responden explícitamente a la cuestión que en este apartado b nos planteábamos en Bailey, Francis y Hill (1987), Gómez, Latorre y Sanjosé (1993) y Salinas y Velazco (1998).

1.c. Dificultades de aprendizaje respecto a la relación de equivalencia entre una espira de corriente y un imán de acuerdo con el marco teórico de la física

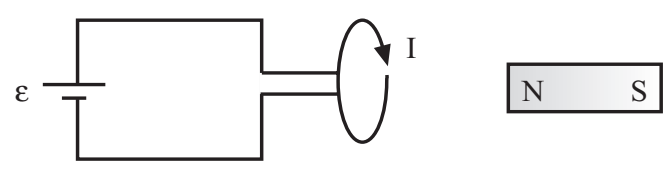

Tenemos una espira fijada en un circuito de corriente continua y enfrente de un imán, como se indica en la figura. ¿Qué sucederá?

Referencia: Almudí (2002)

Comentario: Esta cuestión ha sido contestada por 235 estudiantes de $2^{\circ}$ de bachillerato, $1^{\circ}$ de Ingeniería, $2^{\circ}$ de Ingeniería y $3^{\circ}$ de Físicas. En el supuesto de la figura, lo que se observaría, en un principio, es que la espira y el imán se atraerían. Esto se debería a que el campo magnético que crea la espira actuaría sobre las cargas en movimiento del imán provocando sobre ellas y, en definitiva, sobre el imán una fuerza hacia la espira. Por otro lado, el campo que crea el imán actuaría sobre la corriente que circula por la espira creándose una fuerza sobre ésta de sentido contrario a la anterior. Como se observa desde el punto de vista magnético, la espira de corriente y el imán son equivalentes.

Entre los estudiantes, el porcentaje de respuestas correctas ha oscilado entre el $2 \%$ en $2^{\circ}$ de bachillerato y el $18 \%$ en $3^{\circ}$ de Físicas. Estos pobres resultados sugieren que los estudiantes no relacionan de forma significativa una espira de corriente con un imán como elementos que forman parte de un mismo esquema conceptual. Este resultado es convergente con los resultados expuestos en los apartados anteriores donde hemos visto que la mayoría de los estudiantes no identifican correctamente las fuentes del campo magnético y que además no saben justificar por qué un imán es fuente de dicho campo.

Las respuestas obtenidas para esta cuestión se pueden agrupar básicamente en dos categorías: por un lado, aquéllas que confunden la fuerza con el campo y afirman que el campo que crea la espira tiene el mismo sentido que el del imán y por eso se atraerán; por otro lado, aquellas respuestas que, basándose en recuerdos memorísticos de la inducción magnética, dicen que no habrá interacción al estar el imán y la espira en reposo y sólo si el imán se moviese, se induciría en la espira una intensidad de corriente. 
1.d. Dificultades de aprendizaje en la comprensión de la definición operativa del campo magnético

1.d.1. El diagrama muestra un hilo largo recorrido por una intensidad de corriente $i$ que sale hacia afuera del papel. ¿Cuál sería la dirección del campo magnético en las posiciones $A$ y $B$ ? Elige una de las respuestas que se dan.

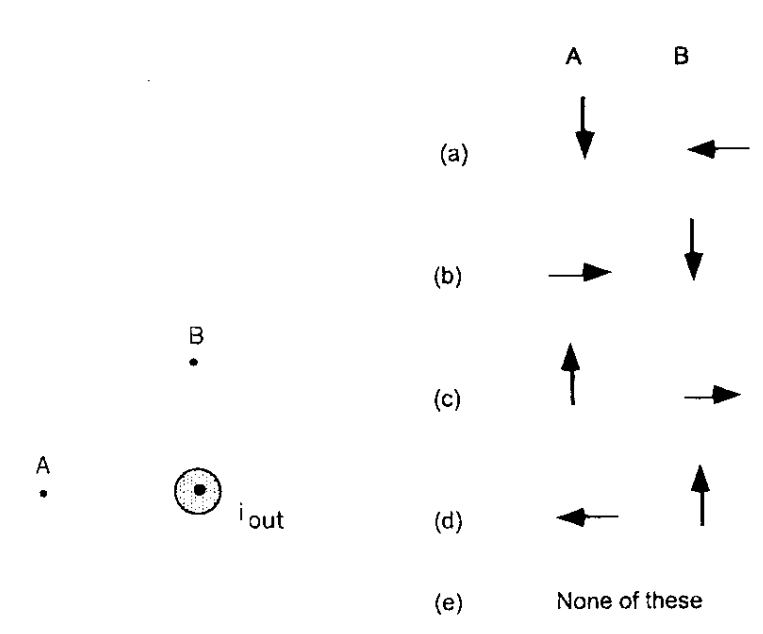

Referencia: Maloney, O'kuma, Hieggelke y Van Heuvelen (2001)

Comentario: Esta cuestión pertenece a un estudio más global que realizaron Maloney y sus colaboradores con una muestra muy extensa de estudiantes norteamericanos, tanto de los últimos cursos de secundaria como de universidad. En ella se observa que los resultados más numerosos en el pretest (las cuestiones se pasaban antes de ver el curso de magnetismo) se correspondieron con la respuesta $b$ con una media del $35 \%$; sin embargo, en el postest la respuesta correcta $(a)$ alcanzaba una media del $56 \%$, cuando en el pretest había sido del $15 \%$. En todo caso, no se puede olvidar que, después de seguir el curso de magnetismo, había todavía un $44 \%$ que respondía incorrectamente siendo las respuestas erróneas, mayoritariamente, la $d$ y la $b$.

Lo anterior indicaría la dificultad de muchos estudiantes para aplicar correctamente la definición operativa del campo magnético. Es posible que también, como indica Maloney, estos resultados, tanto los del pretest como los del postest, apuntarían de alguna forma a que en el pensamiento de los estudiantes se confundiría un hilo recorrido por una intensidad de corriente con un hilo cargado electrostáticamente, es decir, habría una cierta confusión entre el campo eléctrico y el magnético, fundamentalmente en lo que se refiere a sus efectos.

1.d.2. Tenemos dos espiras de corriente idénticas recorridas por una intensidad de corriente $i$. Las espiras están ubicadas como se muestra en le diagrama adjunto. ¿Qué flecha representa la dirección del campo magnético en el punto $P$, punto medio entre las dos espiras?

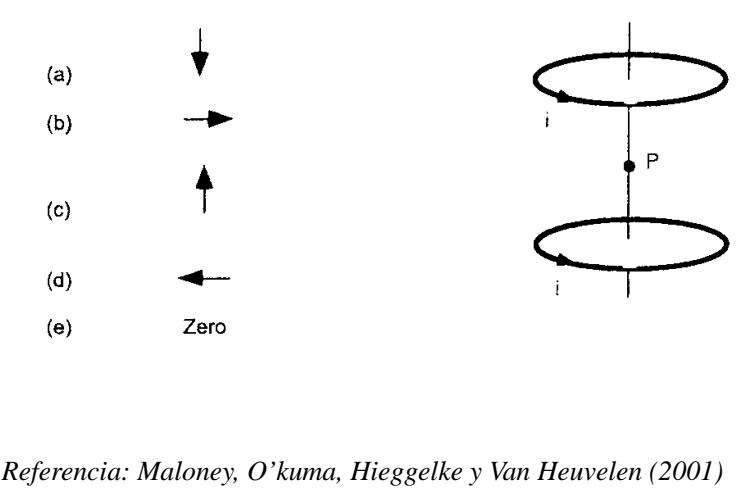

Comentario: Esta cuestión es complementaria de la anterior. Los resultados de la respuesta correcta $(c)$ en el pretest fueron del $20 \%$, pasando después al $47 \%$. En el pretest los resultados más numerosos fueron los de la respuesta e con el $38 \%$ y los de la b con el $22 \%$. En el postest la respuesta $e$ seguía teniendo un porcentaje de respuestas del 33\% y la $b$ del $9 \%$.

Las conclusiones que indican los autores son equivalentes a las explicitadas en la cuestión anterior. En concreto, los autores sugieren que los estudiantes realizarían una analogía entre los campos eléctrico y magnético, en el sentido de considerar como análogos el fenómeno que se presenta en esta cuestión con el que consistiría en calcular el campo eléctrico creado por dos cargas del mismo signo en el punto medio de la recta que las une. Para ello se basan en que, tanto en el pretest como en el postest, hay un $35 \%$ de estudiantes que responde diciendo que la respuesta correcta sería la $e$.

1.d.3. Observa la figura y considera las agujas magnéticas dispuestas en las proximidades de un hilo recorrido por una corriente eléctrica. Escoge la opción correcta que indique el sentido de la corriente:

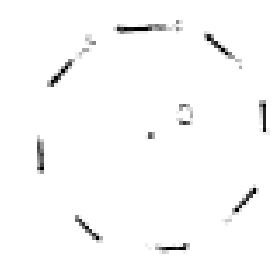

a) Perpendicular a la página en el punto $O$ y orientada de arriba a abajo. b) Paralelo a la página y orientada de izquierda a derecha.

c) Perpendicular a la página en el punto $O$ y orientada de abajo a arriba. d) Paralelo a la página y orientada de derecha a izquierda.

Referencia: Pais de Sousa (1997)

Comentario: La cuestión se enmarca en el trabajo de tesis doctoral de esta investigadora portuguesa y sirve para averiguar si los alumnos conocen el sentido de la corriente rectilínea que determina las líneas del campo magnético representadas. 
El estudio se hace con una muestra de 90 estudiantes, 45 por nivel, pertenecientes al $1^{\circ}$ y $3^{\circ}$ cursos de Ingeniería Industrial.

El porcentaje de alumnos y alumnas que responde correctamente al ítem, contestación $c$, es del $47 \%$ en primer curso y del $45 \%$ en tercero, habiendo respondido a la cuestión prácticamente el $100 \%$ de la muestra de estudiantes. De los resultados, la autora deduce que los estudiantes, incluso de nivel universitario, no conocen comprensivamente la definición operativa del campo magnético, en este caso en lo que se refiere a la dirección y sentido del mismo.

Los lectores podrán encontrar, además, algún trabajo relacionado con el campo magnético y las líneas de campo, que no responde explícitamente a lo que aquí nos planteábamos en Velazco y Salinas (2001).

\section{DIFICULTADES DE APRENDIZAJE RELA- CIONADAS CON LOS EFECTOS DEL CAMPO MAGNETICO: INTERACCIONES MAGNETI- CAS}

En este apartado mostraremos diversas cuestiones que tienen por objetivo investigar las dificultades relacionadas con el aprendizaje no comprensivo de los efectos que produce el campo magnético, es decir, las fuerzas magnéticas; para ello, como ya hicimos en el apartado 1 de este trabajo, nos ha parecido oportuno dividir este apartado en otros más concretos, a saber:

2.a. Dificultades de aprendizaje en detectar sobre qué actúa el campo magnético.

2.b. Dificultades de aprendizaje en relación con la aplicación comprensiva de la ley de fuerza magnética $(\boldsymbol{F}=q \boldsymbol{v} x$ B) tanto desde el punto de vista cualitativo como desde una utilización más cuantitativa.

2.c. Dificultades de aprendizaje en relación con la aplicación de la 3a. ley de Newton en el contexto electromagnético.

2.a. Dificultades de aprendizaje en detectar sobre qué actúa el campo magnético. Además, en ocasiones, confundirán los conceptos de fuerza y campo magnéticos.

2.a.1. ¿Qué le sucede a una carga positiva que se coloca en reposo en un campo magnético uniforme? (Un campo uniforme es aquél en el que el módulo, la dirección y el sentido son los mismos en todos los puntos.)

a) Se mueve con velocidad constante, ya que la fuerza es de módulo constante.

b) Se mueve con aceleración constante, ya que la fuerza tiene módulo constante.

c) Se mueve en un círculo a velocidad constante, ya que la fuerza siempre es perpendicular a la velocidad.

d) Se mueve en un círculo, con una aceleración, ya que la fuerza es siempre perpendicular a la velocidad.

e) Se queda en reposo, ya que la fuerza y la velocidad inicial son cero.

Referencia: Maloney, O'kuma, Hieggelke y Van Heuvelen (2001)
Comentario: La situación planteada, independientemente de la posible aplicación operativa de la ley de la fuerza magnética requeriría reconocer, de una forma comprensiva, un hecho fundamental de la naturaleza como es que los campos actúan exclusivamente sobre las fuentes que los han generado. En este caso, puesto que el campo magnético es engendrado por cargas eléctricas en movimiento, aquél sólo actuará sobre cargas en ese estado de movimiento. De lo anterior parece deducirse que, si los estudiantes no identifican, de una manera significativa, las fuentes del campo magnético estacionario, se van a encontrar con serias dificultades para asumir sobre qué actúa dicho campo.

Concretamente, en el estudio de Maloney y otros la media de respuestas correctas - que era la $e$ - en el pretest fue del $28 \%$, mientras que en el postest sólo subió al $36 \%$. Las demás respuestas quedaron bastante repartidas, lo que conlleva que, incluso después de recibir uno o más cursos de magnetismo, según el nivel de estudios al que se pasaba el cuestionario, había un $64 \%$ de estudiantes que a través de diversas consideraciones llegaban a una conclusión común: el campo magnético actúa sobre cargas que están en reposo.

2.a.2. En la figura se representa un péndulo y un imán. Explica lo que ocurre sobre la bola del péndulo si la bola es de madera y está cargada positivamente.

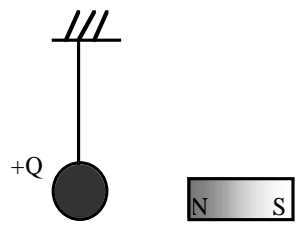

Referencia: Meneses y Caballero (1995)

Comentario: La cuestión ha sido contestada por una muestra de 78 estudiantes del primer curso de Químicas de la Universidad de Burgos. La respuesta correcta, consistente en asegurar que la bola quedaría quieta, ya que el campo magnético del imán no generaría fuerza magnética alguna sobre la bola, ya que ésta se encuentra en reposo, fue muy minoritaria. No se ha valorado, para analizar la respuesta correcta, la polarización eléctrica que la carga $+Q$ produce en el imán y que da lugar a una atracción electrostática entre los dos elementos presentes en la experiencia. De hecho, ningún estudiante lo comentó.

Esta misma cuestión ha sido utilizada en el trabajo de tesis doctoral de uno de los firmantes de este trabajo (Almudí, 2002) y ha sido contestada por una muestra de 70 estudiantes de $2^{\circ}$ de bachillerato, 65 de $1^{\circ}$ de Ingeniería, 60 de $2^{\circ}$ de Ingeniería y 40 de $3^{\circ}$ de Físicas. El porcentaje de respuestas correctas fue del $12 \%, 4 \%, 6 \%$ y $48 \%$ respectivamente.

En ambos estudios, los resultados son convergentes y la respuesta mayoritaria de los estudiantes consiste en indicar que el imán actuaba sobre la bola repeliéndola. El argumento predominante en todos los niveles era que, pues- 
to que el imán en su polo norte (el enfrentado a la bola) es una región cargada positivamente, repelería a la bola cargada con una carga también positiva. Es necesario resaltar que los estudiantes explicaban que la bola era repelida y no que los dos cuerpos se repelían. (Retomaremos este asunto en el apartado 2.c de este mismo trabajo.)

Hubo también otros razonamientos basados en dar entidad real a las líneas de campo que afirmaban que las líneas de campo del imán atraían a la bola. En otros casos era directamente el campo el que ejercía la fuerza.

2.a.3. Una partícula cargada positivamente está en reposo en el plano y está situada entre dos barras de imán tal como se muestra en la figura. El imán de la izquierda es tres veces más poderoso que el de la derecha. ¿Cuál de las flechas que se representa en esa misma figura indica mejor la fuerza ejercida por los imanes sobre la carga?

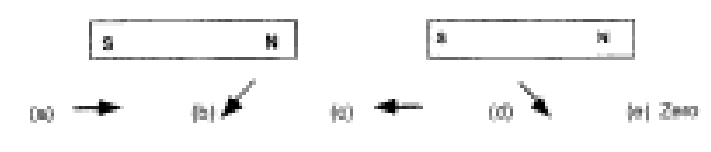

Referencia: Maloney, O'kuma, Hieggelke y Van Heuvelen (2001)

Comentario: Esta cuestión de nuevo trata sobre la posible interacción magnética entre imanes y cargas eléctricas en reposo.

Los resultados obtenidos en el pretest tienen un porcentaje de respuestas correctas de aproximadamente un $31 \%$, pasando a un $35 \%$ después de haber recibido el curso de magnetismo, lo que viene a corroborar una vez más el escaso aprendizaje comprensivo de los estudiantes en lo que se refiere al asunto de sobre qué actúa el campo magnético.

Así pues, a través de las respuestas que se dan para contestar a estas cuestiones, se vuelve a poner de manifiesto el hecho de que los estudiantes no conocen comprensivamente sobre qué actúa el campo magnético. También hemos visto otra serie de dificultades ya observadas como el no-conocimiento de los imanes de forma coherente con el marco de la física y otras que analizaremos más adelante como la no-interpretación correcta de la 3a. ley de Newton.

2.b. Dificultades de aprendizaje en relación con la aplicación comprensiva de la ley de fuerza magnética $(F=q v \times B)$

2.b.1. Indica si estás de acuerdo con la siguiente afirmación: la velocidad de una partícula cargada moviéndose en un campo magnético es siempre perpendicular a la dirección del campo.

Referencia: Bagno y Eylon (1997)

Comentario: En este caso, el porcentaje de respuestas erróneas es del $37 \%$. De ese conjunto de contestaciones, el
$81 \%$ utiliza una recitación de la fórmula arguyendo que $v, B$ y $F$ son siempre perpendiculares de acuerdo con la ley de la mano izquierda o de la derecha del tornillo; el 19\% restante no da razón alguna.

Los autores indican que los resultados ponen de manifiesto que simples reglas nemotécnicas, que en algunos casos son verdad en determinadas condiciones, pasan a tener rango de «ley». Se puede interpretar que los estudiantes responden buscando una solución rápida y segura sin analizar en profundidad las implicaciones reales que desde el punto de vista geométrico se desprenden de la definición de fuerza magnética como el producto vectorial de $q v$ por $B$.

También debemos constatar que, si se plantean en clase la mayoría de los ejercicios bajo la condición de que el campo y la velocidad son perpendiculares, esto podría, en alguna medida, llevar a los estudiantes a la creencia de que ambas magnitudes siempre son perpendiculares.

2.b.2. Un astronauta se encuentra sobre la superficie de la luna y trata de atraer unos clavos con un imán. ¿Lo conseguirá?

Referencia: Bar, Zinn y Rubin (1997)

Comentario: Esta cuestión se pasó a una muestra extensa de estudiantes israelíes de edades comprendidas entre los 9 y los 18 años.

En un $71 \%$ de los casos contestaron que el astronauta no lo conseguiría; los argumentos que dieron, para explicar el fracaso del astronauta, fueron los siguientes:

- Un 29\% dijo que un imán no puede funcionar si no hay aire ni gravedad.

- Un 45\% afirmó que la luna no tiene gravedad y el imán no puede funcionar sin gravedad.

- Un 15\% aseguró que en la luna no hay aire y el imán no puede funcionar sin aire.

- Un 11\% dijo que un imán sólo funciona en la Tierra, ya que la Tierra tiene polos magnéticos.

En este trabajo, los autores tratan de analizar las ideas de los estudiantes acerca de sus ideas previas relativas a la acción a distancia. Llegan a la conclusión de que las ideas de los estudiantes respecto a la fuerza es que actúa a través del contacto; así la acción a distancia necesita de un medio «conductor», como podría ser el aire para que se produzca la gravitación, el magnetismo, etc.

En cuanto a la necesidad de la gravitación para que se produzca el magnetismo (la ciencia dice que no necesita ni el aire ni la gravitación), los autores indican que se podría deber a razonamientos de «sentido común» presentes entre los estudiantes análogos a los de los filósofos griegos, que no distinguían entre gravitación y magnetismo. En todo caso, se constata la dificultad para entender cualitativamente la fuerza magnética. 
2.b.3. Un electrón se mueve horizontalmente hacia una pantalla. El electrón se mueve a lo largo de la trayectoria que se muestra en la figura debido al campo magnético. ¿Cuál será la dirección del campo magnético?

a) Hacia arriba de la página.

b) Hacia abajo de la página.

c) Hacia adentro de la página.

d) Hacia afuera de la página.

e) El campo magnético está en la dirección de la trayectoria curva.

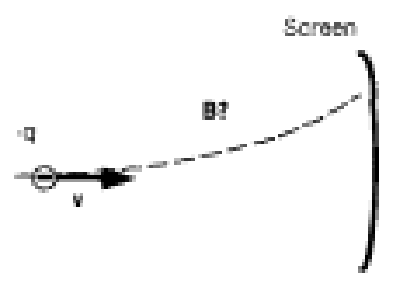

Referencia: Maloney, O’kuma, Hieggelke y Van Heuvelen (2001)

Comentario: Las respuestas correctas a esta cuestión (sería la $d$ ) en el pretest son de aproximadamente el 7\%, pasando al 35\% después de recibir el curso de magnetismo, lo que sugiere que los estudiantes no llegan a asumir las direcciones «no centrales», inherentes a la fuerza y el campo magnéticos y que se diferencian de las de otros campos de la naturaleza como el gravitatorio o eléctrico.

En definitiva, no saben manejar con soltura la operación de producto vectorial que aparece en la fórmula de la fuerza magnética.

Dado que muchas de las respuestas de los estudiantes se corresponden con aquéllas en las que la fuerza se encuentra sobre el plano, ya sea hacia arriba o hacia abajo, los autores de esta cuestión sostienen que esto podría sugerir una cierta confusión entre la fuerza eléctrica y la magnética.

Otras cuestiones analizadas (Anexo, cuestiones III y IV) son coherentes con lo que aquí se ha señalado y muestran, entre otras cosas, que los estudiantes no llegan a asumir las direcciones «no centrales», inherentes a la fuerza y al campo magnéticos.

2.c. Dificultades de aprendizaje en relación con la aplicación de la $3 a$. ley de Newton en el contexto electromagnético.

2.c.1. Dos hilos paralelos I y II, que están cerca el uno del otro, están recorridos por una intensidad de corriente de valor, respectivamente, $i \mathrm{y}$ 3i. Compara las fuerzas que los dos hilos se ejercen entre sí:

a) El hilo I ejerce una fuerza mayor sobre el hilo II que el II sobre el I.

b) El hilo II ejerce una fuerza mayor sobre el hilo I que el I sobre el II.

c) Los hilos se ejercen una misma fuerza de atracción entre sí.

d) Los hilos se ejercen una misma fuerza de repulsión entre sí.

e) Los hilos no se ejercen fuerzas entre sí.

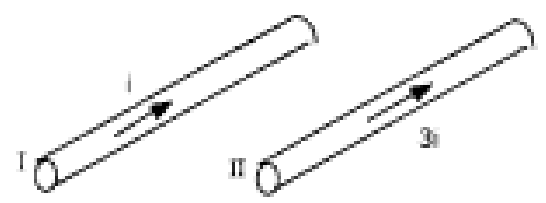

Referencia: Maloney, O'kuma, Hieggelke y Van Heuvelen (2001)

Comentario: Los resultados en esta cuestión para el pretest son que el $48 \%$ de los estudiantes respondía que la contestación correcta era la $b$, mientras que la respuesta correcta, la $c$, sólo era respondida por el $8 \%$. Después de recibir el curso de magnetismo, en el postest, prácticamente el mismo porcentaje seguía diciendo que la respuesta correcta era la $b$, aunque la contestación correcta subía hasta el $24 \%$.

A pesar de los resultados, parece evidente que, para contestar correctamente a esta cuestión, no es necesario un nivel teórico demasiado elevado en magnetismo; por el contrario, saber que los hilos de corriente recorridos por intensidades del mismo sentido se atraen (experiencia de Ampère) y conocer las leyes de Newton impartidas en mecánica es suficiente. De los resultados, sin embargo, se deduce que muchos estudiantes consideran de forma errónea la cuestión que «a mayor intensidad, mayor fuerza» y esto hace que se viole el principio de acción y reacción (la simetría de la fuerza de interacción) que ha sido reiteradamente impartido en mecánica al estudiar las leyes de Newton. El resultado sugiere que los estudiantes no tienen en cuenta lo aprendido en otras partes de la física, en este caso la mecánica, para analizar cuestiones de magnetismo y que, en consecuencia, las dificultades de aprendizaje detectadas en mecánica se extienden a otros campos de la física.

2.c.2. Un hilo recto de corriente se coloca en el campo magnético existente entre los polos de un imán de herradura.

a) Muestra todas las fuerzas que se ejercen sobre todos los componentes del sistema.

b) ¿Se puede aplicar, en este caso, la 3a. ley de Newton?

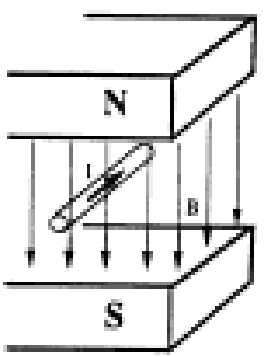

Referencia: Galili (1995)

Comentario: En esta investigación el autor establece la hipótesis de que los posibles obstáculos que se van a presentar podrían originarse como consecuencia de las dificultades que tienen los estudiantes de transferir conceptos 
y leyes de un dominio de la física a otro, en concreto, de la mecánica al electromagnetismo.

La muestra estaba formada por estudiantes de 17-18 años, algunos de grupos de alto nivel de conocimientos, y por futuros profesores de edades comprendidas entre los 23 y los 30 años. Las respuestas mostraron una gran homogeneidad entre todos los grupos y se puso de manifiesto que, al no discurrir los acontecimientos en el ámbito de la mecánica, la 3a. ley de Newton no parece funcionar de la misma forma para los estudiantes. Así, dieron respuestas como: «No estoy seguro, aquí podría no funcionar»; «Nosotros nunca hablamos de eso»; «Esto no es como dos cuerpos empujándose unos a otros»; «Aquí las leyes son diferentes»; o «Es el campo el que empuja al hilo y esto lo que hace que no funcione aquí»». De hecho, sólo un 3\% de los estudiantes mostró una fuerza aplicada sobre el imán, que es de reacción y, por lo tanto, opuesta a la que se ejerce sobre el hilo, que fue la única que se dibujó.

El autor sostiene que la utilización del concepto de campo para calcular fuerzas modifica el modelo y el método para tratar las interacciones. Este cambio, añade, no es explícitamente discutido e incluso mencionado a los estudiantes y futuros profesores. Entiende también que, en mecánica, la interacción se aborda como fuerza entre dos cuerpos y las fuerzas son simétricas, pero que, con el concepto de campo se pierde esa simetría, puesto que el campo no es tratado como un objeto físico (en contra de las ideas de Faraday, Maxwell o la teoría relativista del electromagnetismo que sí lo hace adjudicándole un momento y una energía) y, por lo tanto, la 3a. ley de Newton se torna inaplicable. Parece, pues, que la introducción del concepto de campo enmascara o cuestiona el carácter recíproco de la interacción y esto, seguramente, explicaría las graves dificultades de los estudiantes para aplicar correctamente la 3a. ley de Newton en un ámbito distinto del de la mecánica, como es el caso del magnetismo.

\section{DIFICULTADES DE LOS ESTUDIANTES PARA DISTINGUIR SIGNIFICATIVAMENTE ENTRE EL CAMPO ELECTROSTÁTICO Y EL CAMPO MAGNETTICO ESTACIONARIO}

Como una consecuencia de los déficits constatados hasta ahora, los estudiantes no van a comprender las diferencias entre estos dos campos, fundamentalmente en lo que se refiere a sus efectos. Así, por ejemplo, atribuirán efectos eléctricos a un campo magnético estacionario.

3.1. ¿Cómo explicarías el fenómeno que aparece en la figura adjunta donde un péndulo formado por una hoja de afeitar es atraída por el imán?

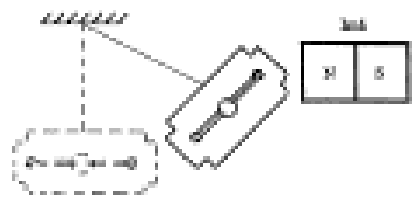

Referencia: Oliveira, De Paulo y Rinaldi (1999)
Comentario: Esta cuestión se pasó a una muestra de estudiantes de 16 años (secundaria) que habían recibido algún curso sobre electromagnetismo y también a un grupo de alumnos y alumnas de primer curso universitario pero que todavía no habían visto ese tema en la universidad.

La respuesta mayoritaria consistió en decir que la atracción se producía como consecuencia de la interacción entre cargas de signo contrario (positiva del imán y negativa de la gilette), atribuyendo, por lo tanto, al campo magnético del imán una connotación de carácter eléctrico, como ya se ha detectado en otras cuestiones que con carácter previo hemos mostrado en este mismo trabajo.

Esto nos podría sugerir que los estudiantes, ante un campo de la naturaleza que básicamente desconocen -el magnético-recurren a otro que creen conocer mejor, como sería el campo eléctrico.

En definitiva, confunden los efectos de los dos campos, no siendo capaces de asumir que el campo magnético del imán ejerce una fuerza sobre los electrones giratorios del material ferromagnético. Esta fuerza hace que el giro de los electrones se oriente en la misma dirección y sentido. La suma de todos estos dipolos microscópicos hace que la hoja de afeitar genere un campo magnético total macroscópico y se comporte como un imán. Así, el imán y la hoja de afeitar se ejercen fuerzas mutuamente y se atraen.

\subsection{Señala la opción correcta:}

Una carga eléctrica positiva, de masa despreciable, se encuentra en reposo próxima al polo Norte de un imán. En estas condiciones, podemos afirmar que la carga:

a) Es atraída por el imán.

b) Es repelida por el imán.

c) No sufre influencia del campo magnético.

Referencia: Pais de Sousa (1997)

Comentario: La mayor parte de los estudiantes desconoce que el campo magnético no influye sobre cargas eléctricas en reposo (como ya hemos visto en apartados anteriores), concretamente el $76 \%$ de los alumnos y alumnas de primer curso universitario y el $82 \%$ de los de tercer curso. Como se ha visto, la mayoría entiende que la carga y el imán van a interactuar (atrayéndose o repeliéndose según el caso: si el imán está enfrentado a la carga por su polo N, repeliéndose, $\mathrm{y}$, si por la cara $\mathrm{S}$, atrayéndose) debido a la interacción electrostática entre la carga y el imán también «cargado» (el polo $\mathrm{N}$ positivamente y el polo $\mathrm{S}$ negativamente). De nuevo parece que se utilizan, sin discriminar, efectos eléctricos y magnéticos.

3.3. Considere dos hilos largos y paralelos separados por una distancia $d$ y recorridos por sendas corrientes eléctricas. Experimentalmente se verifica que hay: a) atracción cuando las corrientes son del mismo sentido; $b$ ) repulsión cuando son de sentido contrario. Elige para el caso $a$ la respuesta que estimes correcta de entre las siguientes:

a.1. La interacción eléctrica es nula y la magnética atractiva.

a.2. La interacción eléctrica es atractiva y la magnética nula.

Elige para el caso $b$ la respuesta que estimes correcta de entre las siguientes: 
b.1. La interacción eléctrica es repulsiva y la magnética, nula.

b.2. La interacción eléctrica es nula y la magnética, repulsiva.

Referencia: Pais de Sousa (1997)

Comentario: Los resultados correctos en esta cuestión fueron escasos: el 20\% en cada uno de los dos cursos universitarios evaluados. La respuesta mayoritaria utilizaba la interacción eléctrica para contestar a esta cuestión.

El argumento básico era que los hilos por el hecho de estar recorridos por una intensidad de corriente generaban campos eléctricos; es decir, la idea alternativa de los estudiantes consiste en creer que un hilo recorrido por una intensidad de corriente no es neutro desde el punto de vista eléctrico. De hecho, muchos afirmaban que los hilos generaban campo eléctricos debido a las cargas que se movían a su través, ya que estaban recorridos por una corriente eléctrica.

Debemos constatar que otras investigaciones, como las desarrolladas por Meneses y Caballero (1995) cuando plantean la cuestión «Qué experiencia diseñarías para demostrar la relación entre los fenómenos eléctricos y magnéticos» y la de Greca y Moreira (1998) cuando plantean «Corrientes del mismo sentido se atraen y de sentido contrario se repelen; cargas estacionarias del mismo signo se repelen y de signos contrarios se atraen. ¿Es esto una paradoja?», obtienen resultados cualitativos convergentes con los resultados de las cuestiones mostradas. Así pues, los trabajos realizados parecen confirmar la confusión de los estudiantes entre campo eléctrico y magnético.

\section{DIFICULTADES PARA APLICAR LAS LEYES DEL MAGNETISMO}

Además de las dificultades que con carácter previo han sido analizadas en este trabajo, debemos hacer notar que, cuando se trata de aplicar determinadas leyes, en el ámbito del magnetismo, la desorientación entre los estudiantes es muy grande; entre otras, esto se cumple en la denominada ley de Ampère.

4.1. Obtener una expresión para el módulo del campo magnético en el interior de un hilo conductor cilíndrico, con corriente $i$. Se puede imaginar este conductor como una concatenación de infinitos hilos de diámetro tendiendo a cero.

Referencia: Greca y Moreira (1998)

Comentario: La cuestión se pasó a 56 estudiantes de Física General II para Ingeniería y la respuesta, por parte de un grupo significativo estudiantes, a la pregunta planteada fue la siguiente:

En el exterior $\rightarrow \mathrm{B}=0$

En el interior $\rightarrow \mathrm{B}=\mu_{0} \mathrm{i} / 2 \pi \mathrm{r}$

Para ello hemos utilizado $\int \mathrm{B} . \mathrm{dl}=\mu_{\mathrm{o}} \mathrm{i}$

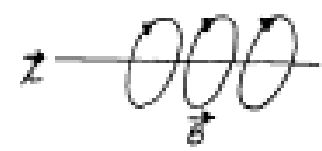

Obsérvese que la respuesta dada es una mera repetición de una figura que aparece en muchos textos, la cual ni siquiera está relacionada con la respuesta, exclusivamente matemática y memorística, que se da al problema. Este grupo de estudiantes, como vieron los autores de esta investigación, presentaba serias dificultades en la resolución de problemas, excepto en aquéllos en que debían aplicar una sola fórmula.

Es evidente que en este problema se podía aplicar la ley de Ampère para resolverlo; al hacerlo se llega a la conclusión de que el cilindro se comporta, para puntos exteriores a él, como un hilo situado en su eje de simetría. Para puntos de su interior, el campo magnético crece con la distancia a medida que me alejo del eje de simetría, ya que la fuente, cantidad de corriente, cada vez es mayor. Parece que los estudiantes simplemente han recordado una fórmula y un dibujo y de forma absolutamente acrítica los han utilizado incorrectamente.

4.2. Sea un hilo de corriente «infinito» por la que circula una intensidad I; dicho hilo de corriente es perpendicular al plano del papel y dirigido hacia afuera (ver figura).

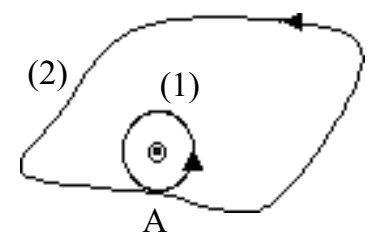

Un estudiante, E1, aplica la ley de Ampère para calcular el campo magnético que crea esa corriente en $A$, único punto de contacto de las dos trayectorias, utilizando la trayectoria circular (1), por cuyo centro pasa el hilo de corriente, y llega a la conclusión de que el valor de dicho campo es: $\mathrm{B}_{\mathrm{A}}=\frac{\mu_{0} \mathrm{I}}{l}$, siendo $l$ la longitud de la circunferencia correspondiente a la trayectoria 1 .

Otro estudiante, E2, hace lo mismo, pero utilizando la trayectoria 2, y

llegando a la conclusión de que el campo magnético en

A vale: $B_{A}=\frac{\mu_{0} I}{L}$, siendo $L$ la longitud de la trayectoria 2 .

Explica, razonadamente, si estás de acuerdo con el estudiante E1, o con el E2, o con los dos, o con ninguno de ellos.

Nota: Las trayectorias 1 y 2 están situadas en el plano del papel.

Referencia: Almudi (2002)

Comentario: El objetivo de esta cuestión era que los estudiantes explicaran qué condiciones se deben dar para que la ley de Ampère se pueda utilizar de una forma rápida y cómoda con el fin de calcular campos magnéticos.

Se considera como respuesta correcta cuando se afirma que tanto el estudiante E1 como el E2 pueden aplicar la ley de Ampère y calcular la circulación del vector «campo magnético», pero sólo el estudiante E1 puede llegar a calcular de forma sencilla y directa el campo magnético. En efecto, utilizando la línea amperiana del estudiante E2, no se puede sacar fuera de la integral de la circulación el campo 
magnético $\mathrm{B}$, ya que no es constante a lo largo de la misma, ni el ángulo que forma con la dirección de cada «elemento diferencial» de la trayectoria es el mismo.

La cuestión se pasó a 165 estudiantes de $1^{\circ}$ y $2^{\circ}$ de Ingeniería y de $3^{\circ}$ de Físicas; el porcentaje de acierto fue, respectivamente, del $20 \%$, $25 \%$ y $38 \%$. Las respuestas erróneas se agruparon en diferentes categorías, siendo una de las mayoritarias aquélla que se denominó «utilización puramente algorítmica de la ley», en la que los estudiantes, de forma acrítica y sin analizar el grado de validez de esta ley, sacaban el campo fuera de la integral y lo despejaban, lo que implicaba que E1 y E2 tenían razón. Otra categoría numerosa es la que se denominó «condición de simetría», consistente en afirmar sin ninguna explicación que la línea amperiana elegida tiene que ser simétrica y, por lo tanto, E2 no tendría razón. Otra categoría menos numerosa fue la catalogada como «coincidencia de la línea amperiana con la de campo» y así decían que E1 tenía razón porque el campo magnético de un hilo son circunferencias y no otro tipo de líneas. Finalmente algunos decían que ninguno tenía razón porque la expresión final debía quedar en función de $r$ (radio de la circunferencia), lo cual sugería que utilizaban un recurso memorístico de la fórmula del campo magnético creado por un hilo de corriente infinito.

Los resultados anteriores junto con otras investigaciones (Kofman y Concari, 2002) sugieren que, para la mayoría de los estudiantes de todos los niveles, el operador circulación del campo no tiene significado y, frecuentemente, lo identifican con el propio campo. Tienden a identificar la circulación del campo magnético a lo largo de una línea cerrada con el campo magnético cuando utilizan la ley de Ampère.

\section{CONCLUSIONES}

El trabajo de recopilación y análisis que se ha hecho en este estudio parece indicar que la mayoría de los estudiantes, tanto de bachillerato como de universidad, encuentran serias dificultades en la comprensión de los conceptos básicos del campo magnético estacionario, en particular los siguientes:

1) No identifican correctamente las fuentes de dicho campo. (Apartados 1a, 1b y 1d)

2) Así mismo, aunque la gran mayoría de los estudiantes reconoce que el imán es fuente del campo magnético, no sabe justificarlo de forma coherente con el marco teórico de la física, no relacionando el comportamiento de un imán con el movimiento de cargas a nivel microscópico. (Apartados $1 \mathrm{~b}$ y $1 \mathrm{c})$

3) En relación con lo anterior, no llegan a establecer una relación de equivalencia, en el ámbito del electromagnetismo, entre una espira de corriente y un imán. (Apartado 1c)

4) Por otro lado, al no establecer con precisión las fuentes del campo magnético estacionario, no reconocen que dicho campo sólo actúa sobre su propia fuente; por lo tanto no comprenden que dicho campo actúa sólo sobre cargas en movimiento. (Apartado 2a)

5) Muchos de ellos no comprenden la diferencia entre el campo electrostático y el campo magnético estacionario, fundamentalmente en lo que se refiere a sus efectos; así, por ejemplo, atribuyen efectos eléctricos a campos magnéticos. (Apartado 3)

6) Los estudiantes encuentran graves dificultades a la hora de establecer una relación cuantitativa entre el comportamiento del campo magnético a lo largo de una línea cerrada y la cantidad de corriente (fuente de dicho campo) que atraviesa la región limitada por la citada trayectoria. (Apartado 4)

7) Incluso, desde el punto de vista operativo, no manejan eficaz y comprensivamente las expresiones matemáticas correspondientes a la fuerza y el campo magnéticos. (Apartados $1 \mathrm{~d}, 2 \mathrm{~b}, 2 \mathrm{c}$ y 4 )

Del análisis de sus respuestas también se ha podido constatar que, además de los déficits de tipo conceptual, que se acaban de señalar, los estudiantes presentaban una serie de dificultades procedimentales que sería necesario abordar desde una enseñanza constructivista. Así pues, un buen conocimiento científico no puede reducirse sólo a tratar aspectos conceptuales, que evidentemente intentarían paliar los déficits apuntados con anterioridad, sino que debe haber, al lado de lo anterior, exigencias procedimentales y, en su caso, axiológicas.

En definitiva, entendemos que todos los aspectos indicados son importantes a la hora de realizar, por parte del profesorado, la secuencia de enseñanza y para concretar de forma precisa la correspondencia entre objetivos y contenidos conceptuales. Evidentemente, dicha secuencia de enseñanza, que debería estar de acuerdo con la investigación en didáctica de las ciencias, tratará de superar las deficiencias que aquí se han puesto de manifiesto. Este ilusionante reto será el objetivo de nuestros próximos trabajos. 


\section{REFERENCIAS BIBLIOGRÁFICAS}

ALMUDÍ, J.M. (2002). «Introducción del concepto de campo magnético en primer ciclo de universidad: dificultades de aprendizaje y propuesta alternativa de orientación constructivista». Tesis doctoral. Universidad del País Vasco.

BAGNO, E. y EYLON, B. (1997). From problem solving to a knowledge structure: An example from the domain of electromagnetism. American Journal of Physics, 65(8), pp. 726-736.

BAILEY, J., FRANCIS, R. y HILL, D. (1987). Exploring ideas about magnets. Research in Science Education, 17, pp. 113116.

BAR, V., ZINN, B. y RUBIN, E. (1997). Children's ideas about action a distance. International Journal of Science Education, 19(10), pp. 1137-1157.

BORGES, A.T. y GILBERT, J.K. (1998). Models of Magnetism. International Journal of Science Education, 20(3), pp. 361378 .

CARRASCOSA, J. y GIL D. (1992). Concepciones alternativas en mecánica. Enseñanza de las Ciencias, 10(3), pp. 314328 .

DRIVER, R. (1989). Student's conceptions and the learning of Science. International Journal of Science Education, 11, pp. 481-490.

DRIVER, R., GUESNE, E. y TIBEGHIEN, A. (1985). Children's ideas in science. Philadelphia: Open University Press.

DUIT, R. y RHÖNECK, C. (1998). Learning and understanding key concepts of electricity, en Tiberghien, A., Jossem, L. y Barojas, J. (eds.). Connecting research in physics education with teacher education, pp. 55-62 (ICPE book 1998).

ERICKSON, G. (1994). Pupils' understanding magnetism in a practical assesment context: the relationship between content, process and progression, en Fensham P., Gunstone R. y White, $\mathrm{R}$. Londres. The content of Science.

FURIÓ, C. y GUISASOLA, J. (1999). Concepciones alternativas y dificultades de aprendizaje en electrostática. Selección de cuestiones elaboradas para su detección y tratamiento. Enseñanza de las Ciencias, 17(3), pp. 441-452.

GALILI, I. (1995). Mechanics background influences students' conceptions in electromagnetism. International Journal of Science Education, 17(3), pp. 371-387.

GIANNETO, E., TARSITANI, C. y VICENTINI MISSONI, M. (1992). The relations between Epistemology, History of Science and Science Teaching from de point of view of the research on mental representations. Proccedings of the Second International Conference on History and Philosophy of Science in Science Education, pp. 359-374. Toronto (Canadá).

GÓMEZ, S., LATORRE, A. y SANJOSÉ, V. (1993). El modelo de Ausubel en la didáctica de la física: una aproximación experimental al proceso de contenidos que presentan constructos poco elaborados por los aprendices. Enseñanza de las Ciencias, 11(3), pp. 235-246.
GRECA, I.M. y MOREIRA, M.A. (1998). Modelos mentales y aprendizaje de física en electricidad y magnetismo. Enseñanza de las Ciencias, 16(2), pp. 289-303.

GUISASOLA, J., ALMUDÍ, J.M. y ZUBIMENDI, J.L. (2003). Dificultades de aprendizaje de los estudiantes universitarios en la teoría del campo magnético y elección de los objetivos de enseñanza. Enseñanza de las Ciencias, 21(1), pp. $79-94$

KOFMAN, H.A. y CONCARI, S.B. (2002). Dificultades conceptuales en la presentación de la ley de Ampère: análisis bibliográfico y propuesta para la enseñanza universitaria. $R e$ vista de Educación en Ciencias, 3(1), pp. 43-46.

MAAROUF, A. y BENYAMNA, S. (1997). La construction des sciences physiques par les représentations et les erreurs: cas des phenomènes magnétiques. Didaskalia, 11, pp. 103-120.

MALONEY, D.P., O'KUMA, T.L., HIEGGELKE, C.J. y VAN HEUVELEN, A. (2001). Surveying students' conceptual knowledge of electricity and magnetism. Phys. Educ. Res., American Journal of Physics Suppl., 69(7), pp. 12-23.

MENESES, J.A. y CABALLERO, M.C. (1995). Secuencia de enseñanza sobre el electromagnetismo. Enseñanza de las Ciencias, 13(1), pp. 36-45.

MILLAR, R. (1996). Investigation des élèves en Science: une approche fondeé sur la connaisance. Didaskalia, 9, pp. 9-30.

OLIVEIRA, J., DE PAULO, S.R. y RINALDI, C. (1999). Investigação do papel da experimentação na construção de conceitos em electricidade no ensino médio. Cadernos Catarinenses de Ensino de Física, 16(1), pp. 105-122.

PAIS DE SOUSA, M.G. (1997). «Forças e campos magnéticos» Tesis doctoral. Universidad de Aveiro.

POZO, J.I. (1999). Más allá del cambio conceptual: el aprendizaje de la ciencia como cambio representacional. Enseñanza de las Ciencias, 17(3), pp. 503-512.

PFUNDT, H. y DUIT, R. (1998). Bibliography: Students' alternative frameworks and science education (6a. ed.) Kiel: Institute for Science Education at the University of Kiel.

SALINAS, J. y VELAZCO, S. (1998). Modelos para el campo eléctrico en estudiantes universitarios a posteriori de la instrucción. Memorias SIEF. IV Simposio de investigadores en educación en Física, pp. 341-351. La Plata (Argentina).

SELMAN, R.L., KRUPA, M.P., STONE, C.R. y JAQUETTE, S (1982). Concrete operational thought and the emergence of the concept of unseen force in children's theories of electromagnetism and gravity. Science Education, 66(2), pp. 181-194.

VELAZCO, S. y SALINAS, J. (2001). Comprensión de los conceptos de campo, energía y potencial eléctricos y magnéticos en estudiantes universitarios. Revista Brasileira de Ensino de Física, 33(3), pp. 308-318.

WANDERSEE, J.H., MINTZES, J.J. y NOVAK, J.D. (1994). Research on alternative conceptions in Science, en Gabel, D.L. (eds.). Handbook of Research on Science Teaching and Learning. Nueva York: Mcmillan Pub. Co. 


\section{ANEXO}

I. ¿Qué es un imán y cómo funciona?

Referencia: Selman, Krupa, Stone y Jaquette (1982)

II. Se da a los estudiantes imanes en diferentes circunstancias (con barreras, sin ellas, etc.) y se les pregunta si atraerán o no a una serie de objetos metálicos.

Referencia: Erickson (1994)

III. Indica si estás de acuerdo con la siguiente afirmación: un campo magnético nunca cambia la velocidad (el módulo de la velocidad) de una partícula cargada que se mueve en él.

Referencia: Bagno y Eylon (1997)
1. Puting Model
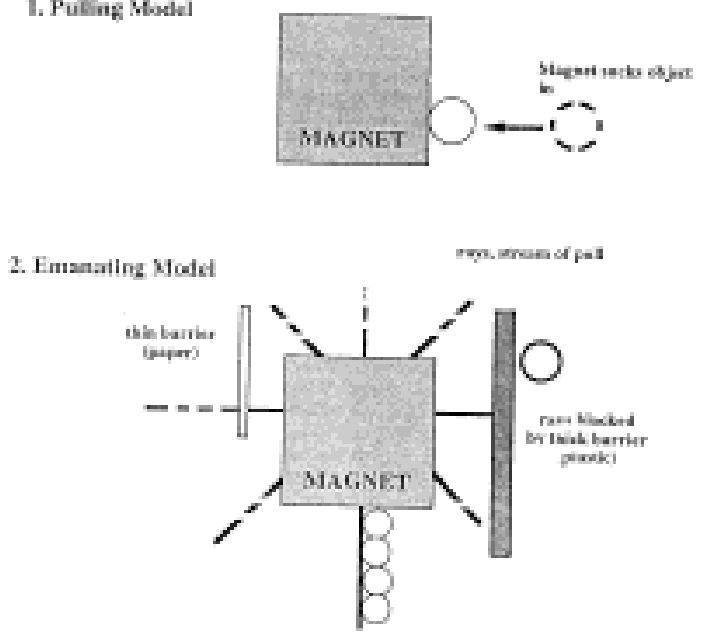

3. Endosing Mnsest

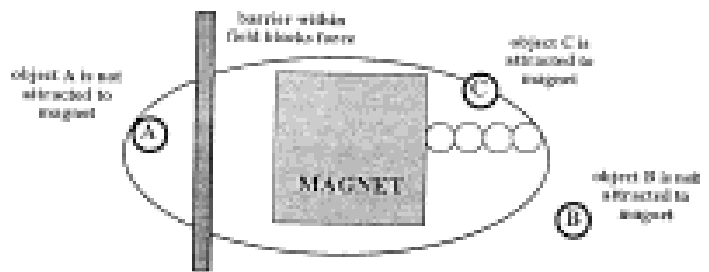

IV. Determina en las figuras el sentido de la fuerza magnética que actúa sobre la carga negativa $q$ haciendo corresponder un número a cada letra y poniéndolo dentro del cuadro.

1) Es nulo

2) Tiene sentido positivo del eje $X$.

3) Tiene sentido positivo del eje Y.

4) Tiene sentido positivo del eje $Z$.

5) Tiene sentido negativo del eje $X$.

6) Tiene sentido negativo del eje Y.

7) Tiene sentido negativo del eje $Z$.

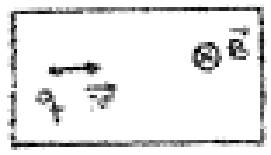

A

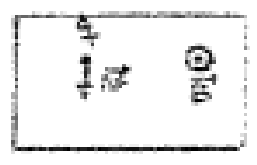

B

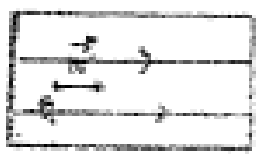

$\mathrm{C}$

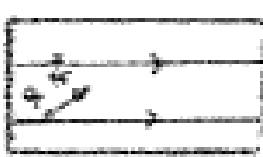

D

Referencia: Pais de Sousa (1997) 